\title{
Green routing in a two-level network of cross docks given the shipping price (Case study: Pegah Co.)
}

\author{
Farhad Bavar*, Majid Sabzehparvar** and Mona Ahmadi Rad* \\ * Department of Industrial Engineering, Islamic Azad University-North Tehran branch, Tehran, Iran \\ ** Department of Industrial Engineering, Collage of Engineering, Karaj Branch, Islamic Azad University, Karaj, Iran \\ **Corresponding Author: sabze@kiau.ac.ir
}

$\begin{array}{ll}\text { Submitted } & : 19 / 03 / 2020 \\ \text { Revised } & : 04 / 05 / 2021 \\ \text { Accepted } & : 11 / 05 / 2021\end{array}$

\begin{abstract}
In this research, a green routing model is provided in a two-level network of cross docking given the shipping price. Three objectives were proposed in this model including: total cost reduction, shipping costs reduction and carbon emissions reduction. The overall objective of the model, is getting the best route in the distribution network which will impose the least cost and also minimizes emissions of environmental pollutants. For the model to approach the real situation, Pegah corporation's warehouses and distribution network are considered as a case study. Solving the developed model was carried out by GAMS. By the size of the problem increasing, the running time of the program is notably increased and this means the problem is NP-hard. So, in order to solve the model in medium and large dimensions, we used meta-heuristic MOGWO and NSGA II algorithm. The results of investigating various problems with meta-heuristics, indicates the high performance of the proposed algorithm in terms of the time needed and the response achieved. Results indicated that the proposed model reduced the emission of environmental pollutants along with total cost and shipping cost reduction. Also given the time window, the products were shipped to customers in a timely manner.
\end{abstract}

Keywords: Cross-docking; Routing; Environmental pollutants; Time windows; Pricing.

\section{INTRODUCTION}

Cross docking is a logistic strategy which aims to reduce inventory and increase customer satisfaction (Buijs et al. 2014). Products are delivered to the customer through cross docking and before shipping the items to the customer they should be collected in cross docks and after that weighing, packing and sorting operations are done based on the destination, and they should be shipped out to customers in the shortest time possible by outgoing vehicles (Dondo Jaime Cerd, 2015). Cross docking mostly acts as an inventory coordinator than a warehouse (Peng-Yeng Yin, Ya- Lan Chuang, 2016). Products are usually stored in cross-docks for less than 24 hours and the cross-dock must be evacuated at the end of the working day (Reddy et al., 2016). Kinnear defined cross-docks as "receiving products from suppliers or manufacturers for various end-users and combining them with other suppliers' products for the ultimate purpose" (Kinnear, 1997). Since cross docks have benefits such as cost savings, supply and delivery reduction, customer service upgrade, storage space reduction, inventory turnover period reduction, inventory 
overload reduction, integration of cargo, resource utilization improvement (for example, using the maximum vehicle capacity), better matching between the shipped product and demand, and its implementation has many benefits (Hasani Goodarzi and Zagardi, 2016; Mozafari, 2021). In supply chain management, cross-docking is a logistic method to minimize warehouse storage and coordinating distribution activities related to loading products between the delivery of vehicles and the carriage of products. There are generally five activities in distribution centers: admission, organizing, temporary maintenance, selection and transfer. Cross-docking operations need to be carefully coordinated due to lack of storage space (Witt, 1998; Vis and Roodbergen, 2008; Miao et al., 2009; Tang and Yan, 2009). On the one hand, vehicle routing problem is the heart of distribution organization. Thousands of companies involved in delivery, collection and transportation of objects and humans, face this problem every day (Hornstra, 2020). Urban logistic service providers always look for ways to deliver goods faster and cheaper than before (Wang et al, 2020). Since organizations have different conditions, the purposes and constraints of this problem are very diverse. Facility capacity is often limited and this limitation can be due to space constraints, limitation of labor etc. However, for some, there is no capacity limitation on the facility (Maknoon te al. 2016). These problems are therefore classified into two categories: problems with limited and problems with unlimited capacity (Amalia et al. 2017). The proposed model has two levels that the first level includes potential cross-docking locations. In addition, the second level includes product customers. In each time period, by minimizing total logistic costs and given the capacity of the vehicles, loading is done between cross docks. This research specifically addresses the following questions:

1) How are time window parameters considered in designing the routing problem?

2) How can we reduce total costs, in vehicle routing problem of the cross-docking network?

3) How can we reduce the emission of environmental pollutants in vehicle routing?

4) How can we find the shortest route for vehicles in cross-docking networks?

Of course, the evidence suggests that to meet customer needs as well as the pressure from international and governmental organizations advocate for environmental problems, organizations have been reacting for many years and have accepted the necessity of environmental management and in order to compete with global markets, they have implemented green supply chain management (Zhu et al. 2010). Many buyer companies demand that their suppliers, implement green supply chain management practices and even carry out additional environmental requirements) (Jabbour, 2009). Moreover, the importance of this issue worldwide is so significan that suppliers are under pressure to find every business opportunity in a new space, without green supply chain management practices (Lee, 2007). An environmental look at the supply chain makes sense in every country, in every industry and on every level. Therefore, according to the material above the purpose of this study is to determine the minimum total shipping costs (distribution cost), and to minimize shipping costs and the emissions of environmental pollutants (carbon). Due to the capacity limitation of the vehicles and the fuel price as well as the problem time window, the fuel price as well as products may have limitations on shipping and that can affect many routing decisions. The most important decision is pricing the products' shipping that has always been accompanied by many challenges and will have different consequences. In this research to carry each unit of products, a certain price is considered. The price is determined based on the route taken by each vehicle. The problem has many complications due to the service being performed at a specific time that is in case of adding to route length limit and time window cost although the complexity rises, but it gets close to a practical topic. According to the above, a time window for truck routing is set. The time window considered for this problem is np-hard, that is vehicles should provide service to customers at a certain interval. Since vehicle, routing is one of the most important components of cross docking network and given the researcher's interest and lack of researches in this area, the researcher has chosen this area. In this model, penalty for keeping inventory is also considered, that is a particular time interval is determined to store products in the warehouse and in case of not evacuating the warehouse a penalty would be considered. Another issue is transportation pricing which is very important given the high fuel prices in the world, is also very practical, and is less researched. There is also a penalty for carbon emission (more than the limit). What follows is a literature review in section 2. Study and formulation are followed in section 3. Section 4 provides a numerical example and optimal solution for the model. In addition, analytical results and sensitivity analysis are discussed. The final results and some suggestions for future research are presented in section 5 . 


\section{LITERATURE REVIEW}

The first study performed in the field of vehicle routing for cross docking was carried out by Lee et al. (2006) to achieve optimized vehicle routing schedules and the model has considered timing and cross-docking routing at the same time and since the problem is NP-hard, it has provided an algorithm based on TS 1to solve the problem. Wen et al (2009) provided the famous article about various Vehicle Routing Problem Cross Docking (VRPCD) considering that products cannot be stored in cross docks and suggested the TS algorithm to solve the model. Moon et al. (2012) provided vehicle routing model and considering the time window, multi-product mode and removal and delivering multiple times. Study and research in the field of pricing problem literature is mainly focused on linear demands with a percentage of error due to persuasive and influential analysis. In the past, research has been specifically conducted on each part of the aforementioned sections, and there are opportunities for further research and development by other researchers. The importance of pricing financial assets has led to the emergence of various theories and models over the last half century. A research titled sustainable design of closed loop supply chain network routing is performed under uncertainty. In this research, considering the economic, social and environmental effects, a new closed-loop model of sustainable inventory routing under uncertainty was presented. In this research, an applied study is solved using meta-heuristic algorithms (Zhalechian et al. 2016). Some research has been done on optimizing the model of vehicle supply chain network under macroeconomic fluctuations. In this study, the designed supply chain network model is solved considering suppliers' selection problem and the problem of shipping and distributing products using TS algorithm (Gao et al., 2015). A multi-objective model is provided to optimize the design of supply chain network based on biogeography under uncertainty. In this study, a new method of two-level optimization is suggested to design a multi-purpose supply chain network (MO-SCND) with unspecified shipping costs and unspecified customer demands. In this study, to solve the model over a large area we used genetic algorithm and for a small area we took advantage of Lingo software. Eventually, an example of a dairy company is provided as a case study to check the applicability of the model (Yang et al. 2015). A multi-objective MBSA meta-heuristic method is provided for designing and planning green supply chain. In the proposed algorithm in this study, the capacity of supply chain entities (factories, warehouses and distribution centers) is scheduled for inventory and flow of material over the planned time horizon. The purpose of the study, is maximizing profit and minimizing environmental impact (Martinez et al. 2015). In another research, an optimizing model is provided for green supply chain management using big data. In this research, three scenarios are provided to improve green supply chain management, and the first optimizing scenario is divided into three sections: first minimizing the risk (and therefore minimizing economic costs); second minimizing carbon emission and the risk and the third one tries to minimize the risk, carbon emission and economic costs simultaneously (Ray et al., 2016). Here are some examples of recent research into vehicle routing:

Wang et al. (2018) in an article titled routing optimization problem in green supply chain stated that with regard to fees and penalties to reduce the use of carbon in transportation, fuel costs have reduced and we'll try to choose places of 1 Tabu search interest close to each other in the supply chain and this will eventually reduce the routes. Forkan et al. (2017) stated in an article titled cross docking vehicle routing that solving the provided model for 20 origins and 50 destinations indicates that the model is efficient and this will reduce transportation costs and optimizes the routes. Amalia et al. (2017) provided an article titled moving products among cross docking pair locations and direct shipping. Peng Ying and Yan Lan (2016) provided an article titled adaptive memory for green vehicle routing with cross docks and the mathematical bee's algorithm was used for this purpose. Bani Amerian et al. (2019) in an article titled heterogeneous routing of profitable vehicles with cross docks stated that paying attention to quick access to products and inventory will optimize the chain problem of interest. Belgrillo et al. (2019) provided a route-based solution approach to the green vehicle routing problem. Reddy et al. (2016) provided a study titled a multi-agent simulation system to provide operations planning and scheduling with multiple cross docks. Table 1 indicates the features of the performed studies. 


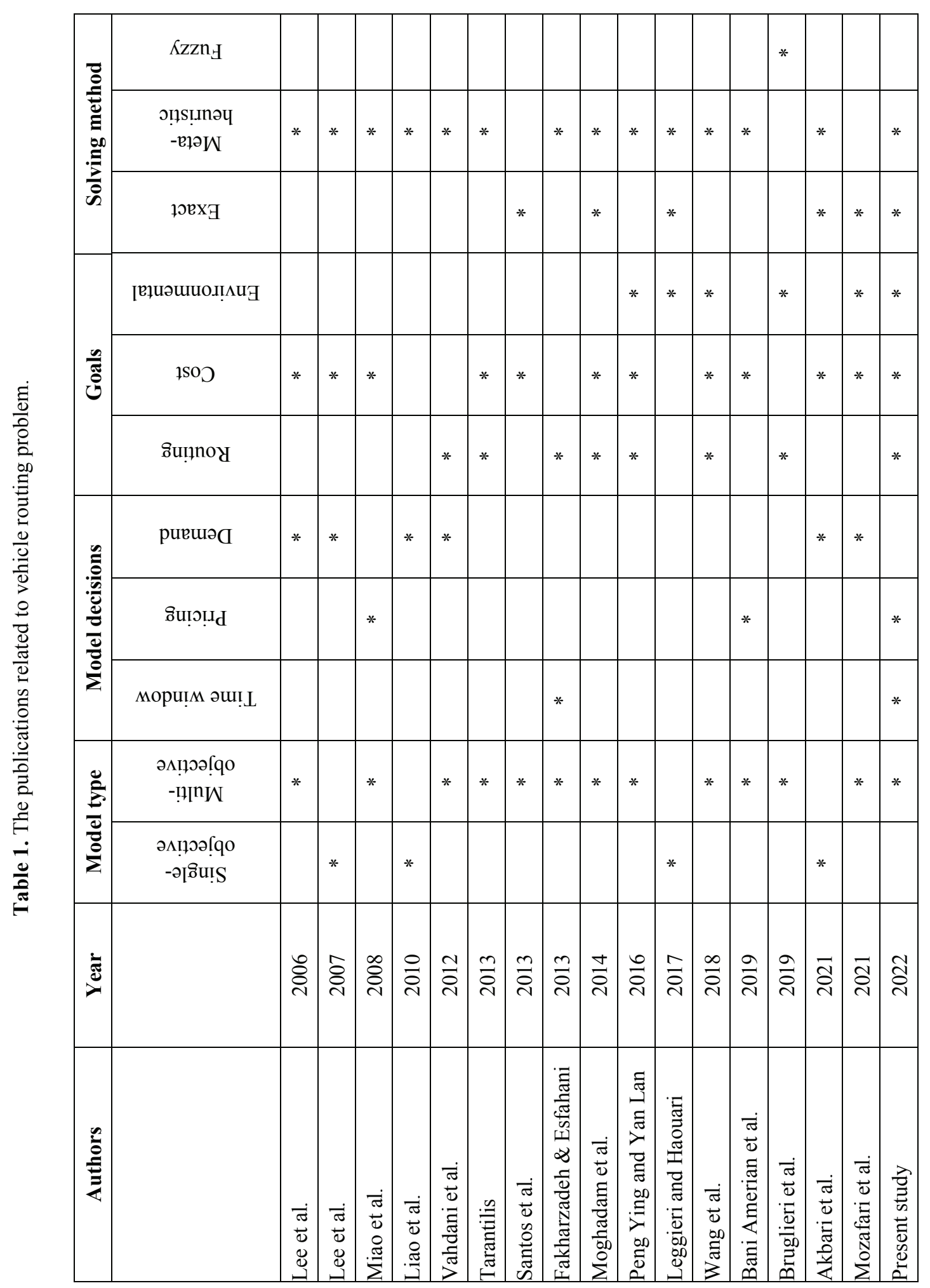


Studying the literature, we can say that, the innovative aspects of this research are:

1) In the proposed model, a specific time frame was determined for storing products in the warehouse that is if the warehouse is not evacuated within that timeframe a penalty will be charged.

2) In majority of the past studies, limited goals such as cost reduction or cost reduction in roads were considered. But there are three contradictory goals in this study including cost, shortest route and reducing environmental pollutants emission.

3) In most the studies carried out, through converting a network to a route and calculating the tolls for the route obtained, the issue has been significantly simplified. But in this study, the provided model is designed over the whole network and for different routes.

4) None of the previous studies considered product shipping pricing, and this research is the first to address this issue.

\section{RESEARCH PROBLEM}

In this research, the routing model is provided for multi-product mode and considers different vehicles, and different routes are provided considering product shipping pricing. Also considering the number of trucks carrying products, the shortest path is selected to reduce the time required. After sorting, products imported to the central cross dock in Tehran, are transferred to vehicles. Then we will consider the shortest route for each of the vehicles to cross docks located in capital cities. After unloading products in warehouses located in capital cities, items are sorted and shipped to the end customers. It is worth noting that some products are directly transferred to vehicles based in cross docks (vehicles are considered to be different that is the vehicle will be unloaded at the end of the route and reaching the cross dock and a new vehicle will carry shipping products to customers).

\section{Hypotheses}

The most important assumptions made in this study are as follows:

- Supply chain includes cross docks and customers

- $\quad$ For each warehouse, there is a minimum and maximum capacity

- The capacity of the central warehouse is limited

- The locations of the warehouses are predetermined.

- The operating time for each vehicle is limited.

- $\quad$ The number of vehicles are limited.

- The capacity of the vehicles is limited.

- Vehicles are capable of carrying one or more specific product types.

- The storage time of the products is limited.

- The route of each vehicle starts from a warehouse but the final warehouse can be different from the initial .

\section{Sets and Indexes}

Numerous signs and parameters have been used in the mathematical model and the definition of each of them is as follows:

$i, j:$ the index for load discharge $(i, j=1,2, \ldots, N)$. ( $\mathrm{i}=0$ indicates the central warehouse in the Tehran).

$v$ : The index for vehicles $(v=1,2, \ldots, V)$ 
$s \quad$ : The index for cross dock $(s=1,2, \ldots, S)$

$\mathrm{N}$ : number of points

$\mathrm{W}$ : a subset of the points

$\mathrm{V}$ : a set of vailable vehicles

$\mathrm{S}$ : number of cross dock units

A : a set of all points and storage units

$\mathrm{M}$ : very large positive number

\section{Parameters}

$\operatorname{COT}_{i j}$ : cost of moving $(i, j)$ Between two nodes of $i, j$

$\mathrm{COT}_{\mathrm{si}}$ : cost of moving $(i, s)$ or the node $(s, i)$ between the cross docks $s$ and customer warehouse $i$

$d_{i} \quad:$ Customer $i^{\text {th }}$ demand

$R_{v} \quad:$ The capacity of the $v^{\text {th }}$ vehicle

$\alpha_{i} \quad$ : The amount of time to start the service for the node $\mathrm{i}$

$\beta_{i} \quad:$ The amount of time the service is delayed for the node $\mathrm{i}$

$E t_{i} \quad$ : The earliest that any service can be started

$Q t_{i} \quad$ : The length of the delivery window at the point of load discharge i

$t_{v} \quad$ : Discharge time from vehicle $\mathrm{v}$

$t_{i, j} \quad$ : Time interval between node load discharge point $\mathrm{i}$ and node $\mathrm{j}$

$d i s_{\mathrm{ij}} \quad$ : Path distance between node load discharge point $\mathrm{i}$ and node $\mathrm{j}$

$\psi_{i} \quad$ : Penalty for violation of unit of time for each node

$E C_{i j} \quad$ : The carbon emission rate from carrying a single commodity from node $\mathrm{i}$ to node $\mathrm{j}$

$E C_{\max }$ : Maximum permissible level of carbon emission

\section{Decision variables}

$V P_{i j v} \quad$ : if vehicle $\mathrm{v}$ passes from interval $(i, j)$ it equals 1 otherwise it will be 0 .

$V P_{\text {siv }}$ : if vehicle $\mathrm{v}$ passes from interval $(s, i)$ it equals 1 otherwise it will be 0 .

$V P_{i s v}$ : if vehicle $\mathrm{v}$ passes from interval $(i, s)$ it equals 1 otherwise it will be 0 .

$U_{i, j} \quad:$ If no vehicle is able to reach its discharge point $i, j$ before the time window is completed it is one, otherwise it is equal to 0 .

$\gamma \quad$ : The penalty for each unit of carbon emitted

$t_{i} \quad$ : Time to get to node i (Service start time for node i)

$w_{i} \quad$ : Extra time spent on node $\mathrm{i}$

$\delta_{i} \quad$ : Service time at node i

$Y_{t} \quad$ : Maximum travel time for vehicles

$\lambda \quad:$ The price of vehicle travel per unit of route 


\section{Mathematical model}

$$
\begin{aligned}
& Z_{1}=\operatorname{Min} \sum_{i=1}^{N}\left[\sum_{\substack{j=1 \\
j \neq i}}^{N} \sum_{v=1}^{V} V P_{i j v} C O T_{i j}+\sum_{s=1}^{S} \sum_{v=1}^{V} V P_{s i v} C O T_{s i}+\sum_{s=1}^{S} \sum_{v=1}^{V} V P_{i s v} C O T_{s i}\right]+\sum_{i=1}^{N} \psi_{i}\left(\alpha_{i}+\beta_{i}\right) \\
& Z_{2}=\operatorname{Min} \sum_{\substack { i=1 \\
\begin{subarray}{c}{j=1 \\
j \neq i{ i = 1 \\
\begin{subarray} { c } { j = 1 \\
j \neq i } }\end{subarray}}^{N} \sum_{v=1}^{V} d i s_{i j} \cdot V P_{i j v} \cdot \lambda \\
& Z_{3}=\operatorname{Min} \sum_{i} \sum_{j} \sum_{v}\left(E C_{i j}-E C_{\max }\right) \cdot V P_{i j v} \cdot \gamma
\end{aligned}
$$

Subject to:

$$
\sum_{i=1}^{N} V P_{i 0 v}=1
$$

$\sum_{v=1}^{\mathrm{N}} \sum_{j=1}^{N} V P_{0 j v}=1$

$\sum_{v=1}^{N} \sum_{i=0}^{N} V P_{i j v}=1$

$\sum_{v=1}^{N} \sum_{j=0}^{N} V P_{i j v}=1$

$\sum_{\substack{j=1 \\ j \neq i}}^{N} \sum_{v=1}^{V} V P_{i j v}+\sum_{s=1}^{S} \sum_{v=1}^{V} V P_{i s v}=1$

$$
\sum_{s=1}^{S} \sum_{v=1}^{V} V P_{s i v}+\sum_{\substack{j=1 \\ j \neq i}}^{N} \sum_{v=1}^{V} V P_{j i v}=1
$$


$\sum_{s=1}^{S} \sum_{i=1}^{N} V P_{s i v}-\sum_{i=1}^{N} \sum_{s=1}^{S} V P_{i s v}=0$

$\sum_{s=1}^{S} \sum_{i=1}^{N} V P_{s i v} d_{i}+\sum_{i=1}^{N} \sum_{\substack{j=1 \\ j \neq i}}^{N} V P_{i j v} d_{j} \leq R_{v}$

$\forall v$

$\sum_{s=1}^{S} V P_{s i v}+\sum_{\substack{j=1 \\ j \neq i}}^{N} V P_{j i v}-\sum_{\substack{j=1 \\ j \neq i}}^{N} V P_{i j v}-\sum_{s=1}^{S} V P_{i s v}=0$

$\forall v, i$

$\sum_{i=1}^{N} \sum_{\substack{j=1 \\ j \neq i}}^{N} V P_{i j v} \leq\left(\sum_{s=1}^{S} \sum_{i=1}^{N} V P_{s i v}\right) * M$

$\forall v$

$\sum_{i=1}^{N} \sum_{j=1}^{N} t_{i j} V P_{i j v}+\sum_{i=0}^{N}\left(\delta_{i}+w_{i}+s_{v}\right) \sum_{j=1}^{N} V P_{i j v} \leq Y_{t}$

$\forall v$

$\sum_{i \in W}^{N} \sum_{\substack{j \in W \\ j \neq i}}^{N} V P_{i j v} \leq|W|-1$

$\forall v \quad \forall W \subseteq A \backslash\{Z\},|W| \geq 2$

$\mathrm{t}_{\mathrm{i}}-Q t_{i} \leq M . U_{i j}$

$\forall i, j$

$\alpha_{\mathrm{i}} \geq E t_{i}-t_{i}$

$\forall \mathrm{i}$

$\beta_{\mathrm{i}} \geq t_{i}-Q t_{i}$

$\forall \mathrm{i}$

$V P_{i j v} \in\{0,1\}$

$\forall i, v \quad i \neq j$

$V P_{\text {siv }} \in\{0,1\}$

$\forall i, v, s$

$V P_{i s v} \in\{0,1\}$

$\forall i, v, s$

$U_{i j} \in\{0,1\}$

$\forall i, j$

$\mathrm{t}_{\mathrm{i}}, \mathrm{w}_{\mathrm{i}}, \sigma_{\mathrm{i}}, Y_{t}, \gamma, \lambda \geq 0$

$\forall \mathrm{i}$ 
Constraint (1) relates to the first objective function. The objective function consists of four parts; including the cost required to travel between points, cost required to travel between the central cross docks and the first points after departure, cost to travel between the last points in each route and the warehouses and finally the cost of not servicing on time should be minimized.

Constraint (2) relates to the second objective function. This objective function is the cost of commodity transportation that should be minimized.

Constraint (3) relates to the third objective function which minimizes the amount of carbon emissions. Constraints (4) to (7) indicate the condition of the vehicle being assigned to the discharge points and the type of goods. Constraints (8) and (9) guarantee that each node is met only once in each time period (Constraint ensures that an edge is traversed by a vehicle if the vehicle that started its route from a cross dock causes a node to be located at the beginning of the path after the warehouse or after another node, and in constraint this path contains the node to node path and the node path to the warehouse and shows that the output from each node can only end up in a warehouse or just another node. Also, these two constraints make service available to all parts of the service). Constraint (10) is about the starting and the ending point of each path that guarantees each path starts from the central warehouse and ends in a warehouse. In other words, they ensure the continuity and continuity of the path. Constraint (11) relates to the capacity of each vehicle that the sum of the points demanded by each vehicle on a route must not exceed the capacity of the vehicle. Constraint (12) makes the input and output of each node equal by each vehicle. And each node receives only one service path. Constraint (13) ensures that an interval can be traversed by a vehicle if it started its journey from a warehouse. Constraint (14) ensures that the service time of each vehicle can not exceed the maximum travel time of that vehicle.

Constraint (15) prevents creating sub travels. Constraint (16) guarantees that no vehicle can reach its destination before the time window. Constraints (17) and (18) relate to the time window. Constraints (18) and (19) are related to the allowable values for model decision variables all of which are zero and one. Constraint (23) also applies to nonnegative variables

\section{SOLVING THE PROPOSED MATHEMATICAL MODEL}

In this section, the results of the numerical solution of the model presented by the exact solution and NSGA II algorithm are presented and they will be validated and compared. First, to validate the proposed modeling, three random samples are produced in small, medium and large sizes. Also, the parameters are randomly generated using a uniform distribution. Then the problems are performed on a laptop by (8GB RAM Intel Core i7) characteristics and software GAMS and linear CPLEX. An epsilon constraint approach is used for the problem to be single objective. Then in problems with low dimensions results of the NSGA II algorithm are compared to the results of the exact model solved by CPLEX of GAMS software and algorithm efficiency will be evaluated. Then, since large-scale exact model resolution is not possible, large-scale problems are solved by NSGA II algorithm. It is worth noting that the genetic algorithm proposed in this study, is coded by MATLAB programming language. Also, beware that to solve problems by the exact algorithm, the time limit is 3600 seconds.

\section{Generating Random Samples}

To solve the model, we implemented the model for 10 experimental problems in different dimensions (small, medium, large), which the size of the designed experiments or in other words the problem size in each experiment is represented in table 2 along with the values of the model input parameters. It should be noted that problems 1 to 3 are considered to be small size, problems 4 to 6 are considered medium size and problems 7 to 10 are considered as large. 
Constraint (15) prevents creating sub travels. Constraint (16) guarantees that no vehicle can reach its destination before the time window. Constraints (17) and (18) relate to the time window. Constraints (18) and (19) are related to the allowable values for model decision variables all of which are zero and one. Constraint (23) also applies to nonnegative variables

\section{SOLVING THE PROPOSED MATHEMATICAL MODEL}

In this section, the results of the numerical solution of the model presented by the exact solution and NSGA II algorithm are presented and they will be validated and compared. First, to validate the proposed modeling, three random samples are produced in small, medium and large sizes. Also, the parameters are randomly generated using a uniform distribution. Then the problems are performed on a laptop by (8GB RAM Intel Core i7) characteristics and software GAMS and linear CPLEX. An epsilon constraint approach is used for the problem to be single objective. Then in problems with low dimensions results of the NSGA II algorithm are compared to the results of the exact model solved by CPLEX of GAMS software and algorithm efficiency will be evaluated. Then, since large-scale exact model resolution is not possible, large-scale problems are solved by NSGA II algorithm. It is worth noting that the genetic algorithm proposed in this study, is coded by MATLAB programming language. Also, beware that to solve problems by the exact algorithm, the time limit is 3600 seconds.

Table 2. Different levels of sample problems.

\begin{tabular}{|c|c|c|c|}
\hline Sample NO & N & V & S \\
\hline 1 & 4 & 5 & 2 \\
\hline 2 & 7 & 10 & 3 \\
\hline 3 & 10 & 20 & 4 \\
\hline 4 & 14 & 35 & 5 \\
\hline 5 & 20 & 50 & 10 \\
\hline 6 & 25 & 70 & 14 \\
\hline 7 & 27 & 80 & 25 \\
\hline 10 & 30 & 90 & 18 \\
\hline 9 & 32 & 100 & 20 \\
\hline
\end{tabular}


Table 3. Input parameters distribution for sample problems.

\begin{tabular}{|c|c|c|c|}
\hline$\alpha_{i}$ & uniform $(0.1,0.2)$ & $\boldsymbol{R}_{\boldsymbol{v}}$ & uniform $(2000,4000)$ \\
\hline$\beta_{i}$ & uniform $(0.3,0.5)$ & $\operatorname{COT}_{i j}$ & uniform $(100,500)$ \\
\hline$E t_{i}$ & uniform $(1,2)$ & $\mathrm{COT}_{S i}$ & uniform $(200,600)$ \\
\hline$Q t_{i}$ & uniform $(5,20)$ & $\psi_{i}$ & uniform $(150,550)$ \\
\hline$t_{v}$ & uniform $(10,20)$ & $E C_{i j}$ & uniform $(10,30)$ \\
\hline$t_{i, j}$ & uniform $(100,300)$ & $d_{i}$ & uniform $(2000,5000)$ \\
\hline$E C_{\max }$ & uniform $(10,15)$ & $d i s_{i, j}$ & uniform $(100,1000)$ \\
\hline$t_{i}$ & uniform $(2,10)$ & $\gamma$ & uniform $(50,100)$ \\
\hline$w_{i}$ & uniform $(10,15)$ & $\lambda$ & uniform $(10,100)$ \\
\hline$\delta_{i}$ & uniform $(5,10)$ & $Y_{t}$ & uniform $(50,500)$ \\
\hline
\end{tabular}

In Table 2, the first column indicates the problem number, the second column represents the total number of points, the third column shows the number of vehicles, and the fourth column shows the number of cross docks. Also, in Table 3, the parameters needed for this problem were randomly generated using a uniform distribution over appropriate time intervals. Now, after generating random samples in different dimensions, problems are implemented in GAMS software and then with the suggested values, we'll execute the problems in different sizes and then the results are provided in the following tables.

\section{The algorithm of Grey Wolf Optimizer}

This algorithm was introduced by Mirjalili et al. In 2014. The method of community leadership, as well as hunting grey wolves, has inspired this optimization algorithm (Rezaei et al., 2017). In order to model the mathematical relations of the chain structure, the best response is considered as the alpha wolf. Also, the second and third optimal responses are considered beta $\beta$ and delta $\delta$, respectively. Other candidate responses are also called omega. In the GWO algorithm, hunting (optimization) is guided by alpha $\alpha$, beta $\beta$ and delta $\delta$. Omega $\omega$ wolves also follow these three wolves in the hope of finding the optimal answer. The mechanism of the GWO algorithm for finding the optimal answer is shown in Figure 1: 


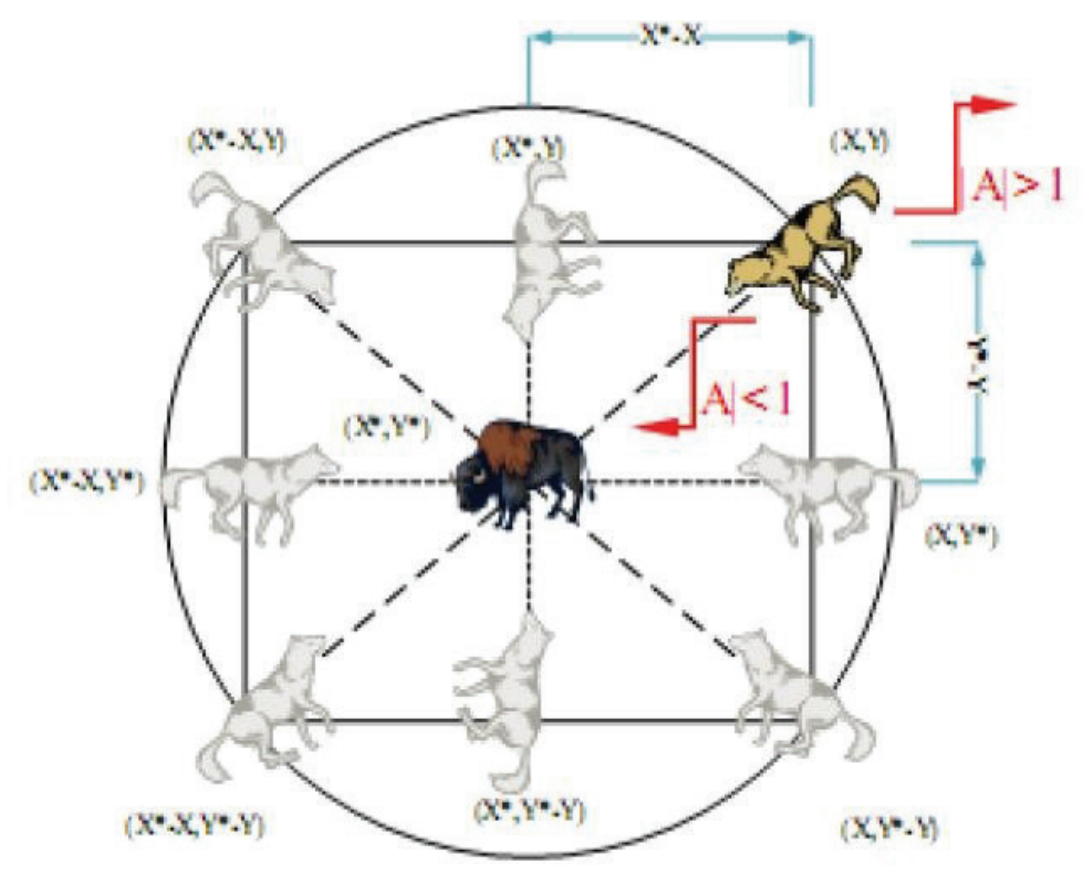

Figure 1. Mechanism of the GWO algorithm to find the optimal solution.

\section{Investigating the Efficiency of the Suggested Algorithms and Solving Sample Problems}

In the proposed Epsilon constraint method, the first objective function is considered as the main objective function and the second function as the secondary objective function; then, 10 breakpoints were considered for the second objective function and a total of 10 Pareto points are generated for each problem. Here, we'll solve five problems designed in the previous sections despite both objective functions by the Epsilon constraint algorithm and the proposed MOGWO algorithm, NSGA II algorithm and the Pareto answers found are reported. To describe the Epsilon constraint method, problem three is chosen as a sample. Problems are first solved separately to determine the Pareto points based on each of the objective functions. The results are reported in table 4.

Table 4. Objective functions optimal values for separate solution.

\begin{tabular}{|c|c|c|c|c|}
\hline Problem No. & Objective type & $\mathbf{Z 1}$ & $\mathbf{Z 2}$ & $\mathbf{Z 3}$ \\
\hline \multirow{3}{*}{3} & Min z1 & 1015 & 88 & 223 \\
\cline { 2 - 5 } & Min z2 & 929 & 69 & 178 \\
\cline { 2 - 5 } & Min z3 & 854 & 54 & 147 \\
\hline
\end{tabular}

After the values of 4 are specified, based on the third step of Epsilon constraint method, 10 Epsilon values are considered for the second objective function. In table 5, Epsilon values of different breakpoints are calculated for the second to third objective functions. The considered breakpoints are also 10. Table 5 shows the values of the breakpoints and the target functions derived at each breakpoint. 
Table 5. Different amounts of epsilons obtained and the values of the objective functions for problem 3.

\begin{tabular}{|c|c|c|c|c|c|}
\hline No. & $\begin{array}{c}\text { Epsilon } \\
\text { value }(\boldsymbol{\varepsilon})\end{array}$ & $\begin{array}{c}\text { Epsilon } \\
\text { value ( } \boldsymbol{\varepsilon} \text { 3) }\end{array}$ & $\begin{array}{c}\text { First } \\
\text { objective }\end{array}$ & $\begin{array}{c}\text { Second } \\
\text { objective }\end{array}$ & $\begin{array}{c}\text { Third } \\
\text { objective }\end{array}$ \\
\hline 1 & 0.1 & 861.91 & 1302 & 94 & 46 \\
\hline 2 & 0.2 & 869.42 & 1280 & 89 & 45 \\
\hline 3 & 0.3 & 876.93 & 1229 & 80 & 45 \\
\hline 4 & 0.4 & 884.44 & 1140 & 78 & 44 \\
\hline 5 & 0.5 & 891.95 & 992 & 66 & 43 \\
\hline 6 & 0.6 & 899.46 & 957 & 64 & 39 \\
\hline 7 & 0.7 & 906.97 & 930 & 53 & 38 \\
\hline 8 & 0.8 & 914.48 & 876 & 48 & 36 \\
\hline 9 & 0.9 & 921.99 & 870 & 40 & 35 \\
\hline 10 & 1 & 929.51 & 861 & 34 & 30 \\
\hline
\end{tabular}

Then, Table 6 shows the Pareto boundary formed by the two algorithms used for problem 3 . As can be seen, NSGA II algorithm and MOGWO algorithm has found nine Pareto answers for problem 3.

Table 6. The optimal Pareto solution obtained from solving No. 3.

\begin{tabular}{|c|c|c|c|c|c|c|c|c|c|}
\hline \multirow{2}{*}{ No. } & \multicolumn{4}{|c|}{ EC } & \multicolumn{3}{c|}{ NSGA II } & \multicolumn{3}{c|}{ MOGWO } \\
\cline { 2 - 11 } & $\begin{array}{c}\text { First } \\
\text { objective }\end{array}$ & $\begin{array}{c}\text { Second } \\
\text { objective }\end{array}$ & $\begin{array}{c}\text { Third } \\
\text { objective }\end{array}$ & $\begin{array}{c}\text { First } \\
\text { objective }\end{array}$ & $\begin{array}{c}\text { Second } \\
\text { objective }\end{array}$ & $\begin{array}{c}\text { Third } \\
\text { objective }\end{array}$ & $\begin{array}{c}\text { First } \\
\text { objective }\end{array}$ & $\begin{array}{c}\text { Second } \\
\text { objective }\end{array}$ & $\begin{array}{c}\text { Third } \\
\text { objective }\end{array}$ \\
\hline 1 & 1302 & 94 & 46 & 1408 & 89 & 43 & 1431 & 91 & 48 \\
\hline 2 & 1280 & 89 & 45 & 1391 & 85 & 42 & 1442 & 89 & 44 \\
\hline 3 & 1229 & 80 & 45 & 1352 & 66 & 35 & 1387 & 73 & 38 \\
\hline 4 & 1140 & 78 & 44 & 1219 & 59 & 41 & 1309 & 65 & 39 \\
\hline 5 & 992 & 66 & 43 & 1211 & 56 & 40 & 1270 & 59 & 37 \\
\hline 6 & 957 & 64 & 39 & 1079 & 43 & 39 & 1155 & 52 & 40 \\
\hline 7 & 930 & 53 & 38 & 1028 & 36 & 37 & 1073 & 42 & 38 \\
\hline 8 & 876 & 48 & 36 & 982 & 29 & 36 & 1020 & 35 & 38 \\
\hline 9 & 870 & 40 & 35 & 912 & 26 & 32 & 952 & 28 & 34 \\
\hline 10 & 861 & 34 & 30 & - & - & - & - & - & - \\
\hline
\end{tabular}


In Figures 2 Pareto points obtained by three algorithms for problem 3 is described as an example with small dimensions.

Objective 1

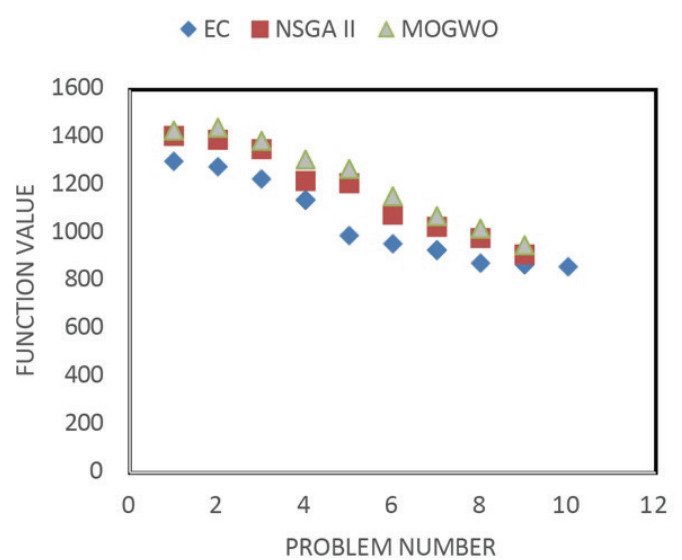

Objective 2

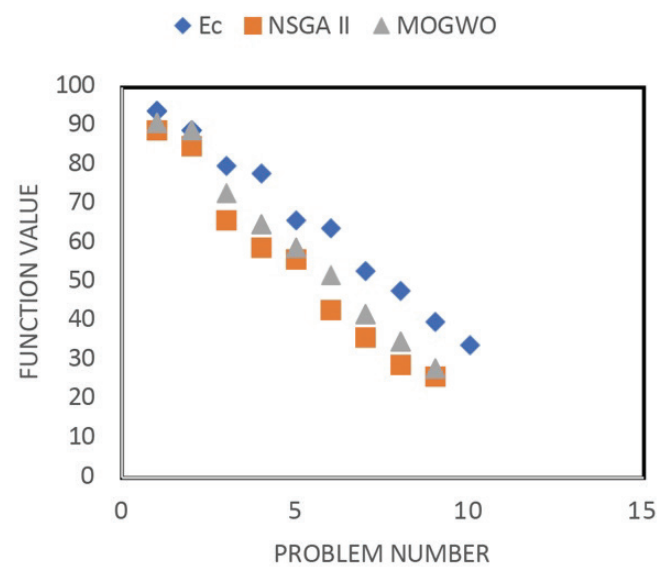

Objective 3

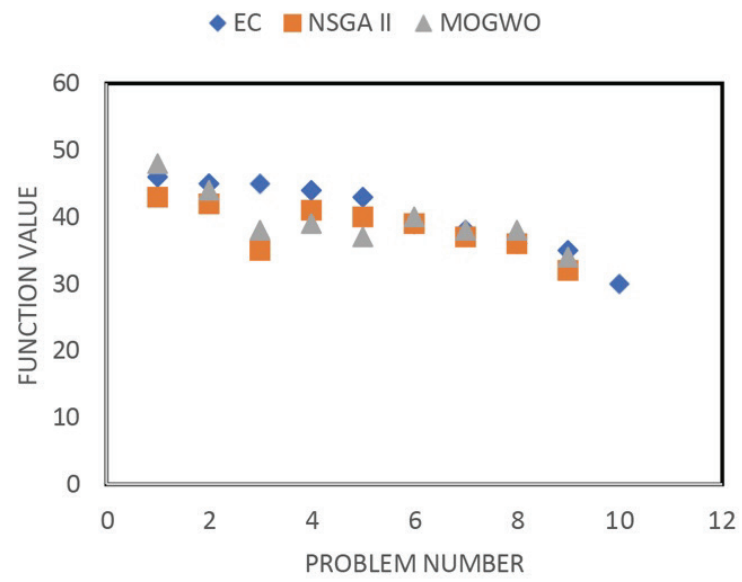

Figure 2. Pareto boundaries created in sample problem 3.

\section{FUNCTIONS}

According to Figures 2, it is obvious that the Pareto boundary proposed by NSGA II algorithm is largely close to the boundary obtained by the MOGWO algorithm. But for more exact validation of the proposed algorithm, and to show to what extent this algorithm is able to identify the optimal Pareto boundary, we use the indices presented in previous sections. For this purpose, three indices MID, SM and DM are calculated and given the SAW values derived from these three indices, we'll investigate the performance of the proposed algorithm. The values calculated for the boundaries obtained by the two algorithms of sample problems 1 to 5 are in accordance with tables 7 to 11 . 
Table 7. Validating algorithms for problem 1.

\begin{tabular}{|c|c|c|c|c|}
\hline Index/methodology & MID & SM & DM & SAW \\
\hline E-constraint & 0.93 & 0.95 & 1.56 & $\mathbf{1 . 2 3}$ \\
\hline NSGA II & 0.97 & 0.98 & 1.22 & $\mathbf{1 . 0 9}$ \\
\hline MOGWO & 0.95 & 1.05 & 1.36 & $\mathbf{1 . 0 4}$ \\
\hline
\end{tabular}

Table 8. Validating algorithms for problem 2.

\begin{tabular}{|c|c|c|c|c|}
\hline Index/methodology & MID & SM & DM & SAW \\
\hline E-constraint & 0.78 & 0.66 & 1.62 & $\mathbf{1 . 4 7}$ \\
\hline NSGA II & 0.81 & 0.73 & 1.47 & $\mathbf{1 . 3 6}$ \\
\hline MOGWO & 0.83 & 0.77 & 1.79 & $\mathbf{1 . 5 1}$ \\
\hline
\end{tabular}

Table 9. Validating algorithms for problem 3.

\begin{tabular}{|c|c|c|c|c|}
\hline Index/methodology & MID & SM & DM & SAW \\
\hline E-constraint & 1.14 & 0.74 & 2.41 & 1.55 \\
\hline NSGA II & 1.15 & 0.82 & 2.21 & 1.43 \\
\hline MOGWO & 1.15 & 0.8 & 2.35 & 1.48 \\
\hline
\end{tabular}

Table 10. Output Results of algorithms for Problem 4.

\begin{tabular}{|c|c|c|c|c|}
\hline Index/methodology & MID & SM & DM & SAW \\
\hline E-constraint & - & - & - & - \\
\hline NSGA II & 1.12 & 0.47 & 1.46 & 1.49 \\
\hline MOGWO & 1.24 & 0.55 & 1.62 & 1.59 \\
\hline
\end{tabular}

Table 11. Output Results of algorithms for Problem 5.

\begin{tabular}{|c|c|c|c|c|}
\hline Index/methodology & MID & SM & DM & SAW \\
\hline E-constraint & - & - & - & - \\
\hline NSGA II & 1.02 & 1.18 & 0.89 & 0.91 \\
\hline MOGWO & 1.05 & 1.21 & 0.95 & 0.98 \\
\hline
\end{tabular}


According to tables 7 to 11 , it is clear that in small dimensions (problems 1 to 3 ), that is until exact solution in 3600 seconds time constraint is possible, NSGA II algorithm performs similar to MOGWO algorithm and Epsilon constraint method and so it can be a good tool to solve this problem in times of inefficiency. For example, from problem 4 onwards, Epsilon constraint method is not able to resolve problems within the time limit specified. Therefore, considering the appropriate performance of NSGA II algorithm similar to MOGWO algorithm, this algorithm is used to solve high-dimensional problems.

\section{CONCLUSIONS}

In this study, a green routing mathematical model was studied in a two-level cross-docking network considering the shipping price (case study: Pegah Co.). The objectives of the problem include total cost reduction, transportation cost reduction and reduction of environmental pollutants emission. Total cost includes the cost required to travel between points, cost to travel the routes between central cross dock and the first points after transportation, and the cost required to travel between the last points on each route and the warehouses and ultimately, the cost of not being able to service them on time is minimized. The proposed problem was solved by GAMS software. Since it was timeconsuming to solve large-scale problems with this software, we provided the similar to MOGWO and NSGA II algorithms. To evaluate the proposed algorithms in small dimensions, we compared its responses to GAMS solution and for medium to large dimensions, we'll obtain the objective function in 10 iterations for the algorithms and then compare them. And we tested the efficiency of the proposed model and the applicability of the algorithms for various problems. The computational results showed that although both methods are suitable both temporally and in terms of objective function values, but the genetic algorithm is more efficient than the exact method.

Finally, some suggestions were proposed for developing the chain structure, parameter development, and developing evaluation criteria for those interested in the area. In the proposed model, central cross-docks as well as capital warehouses are jointly considered. A cooperative game can be used to solve this model. In the proposed model the focus was on transportation of goods which has been addressed in subsequent research on other levels of the supply chain. The proposed model has three outcomes, the first seeks to reduce costs, the second seeks to reduce the price of freight, and finally, it seeks to reduce emissions. In the future, we may consider other evaluation criteria such as supply chain reliability, customer satisfaction fluctuations and etc. The proposed model is a periodic one. Researchers can extend the model to several periods. Future research can also take into account personnel costs, including employees and drivers.

\section{REFERENCES}

Akbari, F.J., Hafezalkotob, A. 2021. Robust cooperative planning of relief logistics operations under demand uncertainty: a case study on a possible earthquake in Tehran. International Journal of Systems Science: Operations \& Logistics, DOI: 10.1080/23302674.2021.1914767

Bruglieri, M., Mancini, S., Pezzella, F., Pisacane, O. 2019. A path-based solution approach for the Green Vehicle Routing Problem. Computers \& Operations Research 103: 109-122.

Buijs, P., Vis, I.F.A., Carlo, H. J. 2014. Synchronization in cross-docking networks: A research classification and framework. European Journal of Operational Research 239: 593- 608. doi:10.1016/j.ejor.2014.03.012.

Dondo, R., Cerdá, J. 2015. The heterogeneous vehicle routing and truck scheduling problem in a multi-door crossdock system. Computers \& Chemical Engineering 76: Pages 42-62. 
Fakhrzadeh, M., Esfahani, A.S. 2013. Modeling the Time Windows Vehicle Routing Problem in Cross-docking 38 Strategy Using Two Meta-Heuristic Algorithms. International Journal of Engineering-Transactions A: Basics, 27. 1113

Guo, C., Liu, X., Jin, M., Lv, Z. 2016. The research on optimization of auto supply chain network robust model under macroeconomic fluctuations. Chaos, Solitons \& Fractals 89: 105-114.

Hornstra, R.P., Silva, A., Roodbergen, K.J., Coelho, L.C. 2020. The vehicle routing problem with simultaneous pickup and delivery and handling costs. Computers \& Operations Research 115: Article 104858.

Jabbour, A., Gao, L., Kwan, J., Watson, A., Liu, X., Zhou, M.D., Graham, R.M., Hicks, M., Macdonald, P.S. 2009. A Recombinant Human Neuregulin-1 Peptide Added to Celsior Solution Further Improves Preservation of the Transplanted Rat Heart after Prolonged Hypothermic Storage. The Journal of Heart and Lung Transplantation 28(2) Supplement, Page S150.

Kinnear E., 1997. Is there any magic in cross-docking. Supply Chain Management: An International Journal 2(2):49- 52 .

Lee, D., Dong, M., 2007. A heuristic approach to logistic network design for end-of-lease computer products recovery.

Transportation Research part E 44:455-74.

Lee, Y.H., Jung, J.W., Lee, K.M. 2006. Vehicle routing scheduling for cross-docking in the supply chain. Computers \& Industrial Engineering 51: 247-256.

Leggieri, V. \& Haouari, M. 2017. A practical solution approach for the green vehicle routing problem. Transportation Research Part E: Logistics and Transportation Review 104:97-112, August 2017.

Liao, C.J., Lin, Y., Shih, S.C. 2010.Vehicle routing with cross-docking in the supply chain. Expert Systems with Applications 37:6868-6873.

Lin, C., Choy, K.L., Ho, G.T.S., Chung, S.H. and Lam, H. Y. 2014. Survey of Green Vehicle Routing Problem: Past and future trends. Expert System with Application 41(4):1118-1138.

Maknoon, M.Y. Soumis, F. Baptiste, P. 2016. Optimizing transshipment workloads in less-than-truckload crossdocks, International Journal of Production Economics 179: 90-100.

Miao, Z., Fu, K., Fei, Q., Wang, F. 2008. Meta-heuristic algorithm for the transshipment problem with fixed transportation schedules. In: New frontiers in applied artificial intelligence. Lecture Notes in Artificial Intelligence 5027:601-10.

Moghadam, S.S., Ghomi, S.F., Karimi, B. 2014. Vehicle routing scheduling problem with cross docking and split deliveries. Computers \& Chemical Engineering 69: 98-107.

Moon, I., Lee, Jeong, H., Seong, J. 2012. Vehicle routing problem with time windows considering overtime and outsourcing vehicles. Expert Systems with ApplicationsVolume 39(1815):13202-13213.

Nikolopoulou, A. I., Repoussis, P.P., Tarantilis C.D., Zachariadis, E. E. R. 2017. Moving products between location pairs: Cross-docking versus direct-shipping. European Journal of Operational Research 256(3): 80819

Pan, S., Nigrelli, M., Ballot, E., Sarraj, R., Yang, Y. 2015. Perspectives of inventory control models in the Physical Internet: A simulation study. Computers \& Industrial Engineering 84: 122-132.

Peng-Yeng, Y. \& Ya-Lan, C. 2016. Adaptive memory artificial bee colony algorithm for green vehicle routing with cross- docking. Applied Mathematical Modelling 40(21-22): 9302-9315.

Ray, Y., Zhong, S.T., Newman, G.Q, Huang, S.L. 2016. Big Data for supply chain management in the service and manufacturing sectors: Challenges, opportunities, and future perspectives. Computers \& Industrial Engineering 101: 572-591. 
Reddy, R. H., Kumar, S.K., Fernandes, K. J., Tiwari, M.K. 2016. A Multi-Agent System based simulation approach for planning procurement operations and scheduling with multiple cross-docks. Computers \& Industrial Engineering 107, May 2017, Pages 28900.

Rezaei, H., Bozorg-Haddad, O., Chu , X. 2018. Grey Wolf Optimization (GWO) Algorithm. In: Bozorg-Haddad O. (eds) Advanced Optimization by Nature-Inspired Algorithms. Studies in Computational Intelligence 720. Springer, Singapore. https://doi.org/10.1007/978-981-10-5221-7_9

Santos, F.A., Geraldo, R.M., Cunha, A.S. 2013. The pickup and delivery problem with cross-docking. Computers \& Operations Research 40: 1085-1093.

Tarantilis, C.D. 2013. Adaptive multi-restart TS algorithm for the vehicle routing problem with crossdocking,

Optimization Letters 7: 1583-1596.

Vahdani, B., Tavakkoli-Moghaddam, R., Zandieh, M., Razmi, J. 2012. Vehicle routing scheduling using an enhanced hybrid optimization approach. Journal of Intelligent Manufacturing 23: 759-774.

Hafezalkotob, A., Mozafari, P. 2021. Hazardous infectious waste collection and government aid distribution during COVID-19: A robust mathematical leader-follower model approach. Sustainable Cities and Society 102814. DOI: $10.1016 /$ j.scs.2021.102814

Mozafari, P\& Valizadeh, J.. 2021. A novel cooperative model in the collection of infectious waste in COVID-19 pandemic.

Journal of Modelling in Management. Vol. ahead-of-print No. ahead-of-print., DOI:10.1108/JM2-07-20200189.

Sadeh, E., Amini, Z. A., Hafezalkotob, A. 2020. Robust optimization model for sustainable supply chain for production and distribution of Polyethylene pipe. Journal of Modelling in Management 15(4): 1613-1653.

Vis, I.F.A. \& Roodbergen, K.J., 2008. Positioning of goods in a cross-docking environment. Computers \& Industrial Engineering 54: 677-689.

Wang, S., Tao, F., Shi, Y. 2018. Optimization of Location-Routing Problem for Cold Chain Logistics Considering Carbon Footprint. International Journal of Environmental Research and Public Health 15(1): 86. doi: 10.3390/ijerph15010086.

Wen, M., Larsen, J., Clausen, J., Cordeau, JF., Laporte, G. 2009. Vehicle routing with cross-docking. Journal of the Operational Research Society 60(12):1708-18.

Witt, CE., 1998. Crossdocking: concepts demand choice. Material Handling Engineering 53(7) : 49.

Yan, H., Tang, Sl. 2009. Pre-distribution and post-distribution cross-docking operations. Transportation Research Part E: Logistics and Transportation Review 45(6):843-59.

Yang, G.Q., Liu, Y.K., Yang, K. 2015. Multi-objective biogeography-based optimization for supply chain network design under uncertainty. Computers \& Industrial Engineering 85: 145-156.

Zhalechian, M. et al. 2016. Sustainable design of a closed-loop location-routing-inventory supply chain network under mixed uncertainty. Transportation Research Part E: Logistics and Transportation Review 89: 182-214.

Zhu, Q., Geng, Y., Lai, K.H. 2010. Circular economy practices among Chinese manufacturers varying in environmental- oriented supply chain cooperation and the performance implications. Journal of Environmental Management 91( 6): 1324-1331. 